\title{
H3C13 Gene
}

National Cancer Institute

\section{Source}

National Cancer Institute. H3C13 Gene. NCI Thesaurus. Code C154647.

This gene is involved in DNA compaction. 